\title{
The third-order law for magnetohydrodynamic turbulence with shear: Numerical investigation
}

\author{
M. Wan, ${ }^{1}$ S. Servidio, ${ }^{1,2}$ S. Oughton, ${ }^{3}$ and W. H. Matthaeus ${ }^{1}$ \\ ${ }^{1}$ Department of Physics and Astronomy and Bartol Research Institute, University of Delaware, \\ Newark, Delaware 19716, USA \\ ${ }^{2}$ Dipartimento di Fisica, Universita' della Calabria, I-87036 Cosenza, Italy \\ ${ }^{3}$ Department of Mathematics, University of Waikato, Hamilton 3240, New Zealand
}

(Received 3 March 2010; accepted 25 March 2010; published online 12 May 2010)

\begin{abstract}
The scaling laws of third-order structure functions for isotropic, homogeneous, and incompressible magnetohydrodynamic (MHD) turbulence relate the observable structure function with the energy dissipation rate. Recently [Wan et al. Phys. Plasmas 16, 090703 (2009)], the theory was extended to the case in which a constant velocity shear is present, motivated by the application of the third-order law to the solar wind. We use direct numerical simulations of two-dimensional MHD with shear to confirm this new generalization of the theory. The presence of the shear effect broadens the circumstances in which the law can be applied. Important implications for laboratory and space plasmas are discussed. (C) 2010 American Institute of Physics. [doi:10.1063/1.3398481]
\end{abstract}

\section{INTRODUCTION}

A very important result in the statistical description of turbulent flows is the so-called four-fifths law for the thirdorder structure function. ${ }^{1,2}$ The $4 / 5$ law has been widely used in the study of turbulence and is fundamental in characterizing the turbulent energy cascade in incompressible hydrodynamics (HD). ${ }^{3}$ The magnetohydrodynamic (MHD) analog of the 4/5 law was derived by Politano and Pouquet, ${ }^{4,5}$ for the case of steady, incompressible homogeneous isotropic MHD turbulence. When the underlying assumptions of the thirdorder law are satisfied, it offers a powerful tool for examining the cascade rate, a fundamental parameter in any application of turbulence theory. Accordingly a number of recent studies of interplanetary turbulence ${ }^{6-9}$ have employed the MHD third-order law to characterize local cascade and heating rates, as an alternative to estimates of energy decay rates based on large-scale energy and correlation scales. ${ }^{10-12}$ It was recently pointed out ${ }^{13}$ that the third-order law can be modified for MHD when a large-scale constant velocity shear is present, in analogy to similar effects that occur for homogeneous shear in $\mathrm{HD} .{ }^{14}$ Here we examine in greater detail the effects of large-scale shear in MHD on the thirdorder law, in both its original form and in the modified form obtained when shear is separated from fluctuations in the sense of a Reynolds decomposition.

The third-order law for isotropic HD identifies the inertial range of the energy cascade and is given by

$$
\left\langle\left(\delta u_{L}(\mathbf{x}, \mathbf{r})\right)^{3}\right\rangle=-\frac{4}{5} \epsilon r,
$$

where $\delta u_{L}(\mathbf{x}, \mathbf{r})=[\mathbf{u}(\mathbf{x}+\mathbf{r})-\mathbf{u}(\mathbf{x})] \cdot \hat{\mathbf{r}}$ is the longitudinal velocity increment along the spatial variation $\mathbf{r}$, and $\langle\cdots\rangle$ indicates an ensemble average. The constant $\epsilon$ is the mean energy dissipation rate, assumed to remain finite as the Reynolds number increases without bound.

For $\mathrm{MHD}^{4-6}$ the third-order law implies two symmetric scaling laws in terms of Elsässer fields $\mathbf{z}^{ \pm}=\mathbf{v} \pm \mathbf{b}$. For full isotropy and homogeneity, the following laws are obtained:

$$
\left\langle\delta z_{L}^{\mp}\left|\delta z_{i}^{ \pm}\right|^{2}\right\rangle=-\frac{4}{d} \epsilon^{ \pm} r,
$$

where $d$ is the spatial dimension, $\delta \mathbf{z}^{ \pm}=\mathbf{z}^{ \pm}(\mathbf{x}+\mathbf{r})-\mathbf{z}^{ \pm}(\mathbf{x})$ are the increments of the Elsässer variables, and $\epsilon^{ \pm}$are the mean energy dissipation rates of the corresponding variables $\mathbf{z}^{ \pm}$.

A number of subtleties arise concerning the exact assumptions that can lead to Eq. (1) (Ref. 15) and, analogously, in Eq. (2). The essential point is that homogeneity, time stationarity, and isotropy must be satisfied, in some form, in order to arrive at the law in the above form of Eqs. (1) and (2). These constraints are rarely verified in nature, so a generalization of this important law of turbulence is needed. Regarding the isotropy constraint, in the HD case Casciola et $a l .{ }^{14}$ extended the $4 / 5$ law to shear-dominated flows, finding that additional terms enter the balance between dissipation and the third-order structure function. Their approach assumes that the shear is a coherent large-scale field, distinct from the fluctuations in that the latter are better described by a statistical approach rather than a detailed description of each realization. In contrast, the large-scale flows, including the shear, are assumed to be prescribed. This suggests a Reynolds decomposition, ${ }^{16}$ in which the velocity field $\mathbf{v}$ can be written as $\mathbf{v}(\mathbf{x}, t)=\mathbf{U}(\mathbf{x})+\mathbf{u}(\mathbf{x}, t)$, where $\mathbf{U}$ is the mean part and $\mathbf{u}$ is the fluctuation part of the total velocity. The existence of nonuniform $\mathbf{U}$ can introduce anisotropy to the flow and the simplest case, where $\nabla \mathbf{U}$ is a constant matrix, has been considered in Ref. 14. The case of large-scale coherent shear has an interesting counterpart in the MHD case. The details of the implications for the MHD third-order law when the velocity shear is constant have been given recently ${ }^{13}$ and will be reviewed below. Here we examine the extended MHD law using direct numerical simulations of twodimensional (2D) MHD turbulence. Several analysis approaches are employed to improve understanding of the physical meaning of the third-order law with shear. An appreciation of these effects and an understanding of how to deal with shear in this context may be of practical impor- 
tance in analysis of MHD turbulence datasets where shear may be present. For example, in the solar wind, ${ }^{6-9}$ largescale shear can occur in connection with the interfaces between high-speed and low-speed streams. ${ }^{17}$

The outline of the paper is as follows: In Sec. II, the third-order law for sheared MHD is briefly reviewed. The 2D MHD equations, together with the numerical procedure, are presented in Sec. III. In the same section, results of isotropic (no shear) and anisotropic (with shear) turbulence simulations will be compared. Initially Reynolds decomposition is employed, both in deriving the appropriate third-order law (Sec. II) and in the data analysis (Sec. III). Subsequently in Sec. IV, we show the theory and data analysis that obtain without use of Reynolds decomposition. Finally, the summary and discussions are given in Sec. V.

\section{THE THIRD-ORDER LAW FOR 2D MHD WITH CONSTANT SHEAR}

Following procedures analogous to those used to obtain the isotropic MHD 4/5 law, ${ }^{4-6}$ and the HD extension to the 4/5 law for constant shear, ${ }^{14}$ one can obtain an MHD thirdorder law with constant shear of velocity. ${ }^{13}$ This is based on the assumption that the velocity shear is not a statistical quantity, and therefore one adopts a decomposition of the velocity field $\mathbf{v}=\mathbf{U}+\mathbf{u}$ into a mean velocity $\mathbf{U}(\mathbf{x})$ and a fluctuating component $\mathbf{u}(\mathbf{x}, t)$. In this way, we arrive at the modified third-order law for MHD in integral form,

$$
\begin{aligned}
& \oint\left[\left\langle\left(\delta z_{k}^{\mp}+\delta U_{k}\right)\left|\delta z_{i}^{ \pm}\right|^{2}\right\rangle n_{k}\right] \mathrm{d} S_{r}+2 \frac{\partial U_{i}}{\partial x_{k}} \int\left\langle\delta z_{i}^{ \pm} \delta z_{k}^{\mp}\right\rangle \mathrm{d} V_{r} \\
& =-4 V \epsilon^{ \pm}
\end{aligned}
$$

Here $\mathrm{d} S_{r}$ and $\mathrm{d} V_{r}$ are the surface and volume elements, $n_{k}$ is a unit vector normal to the surface, $V$ is the actual volume of integration, and the mean energy dissipation rates are $\epsilon^{ \pm}$ $\equiv \nu\left\langle\left|\partial_{k} z_{i}^{ \pm}\right|^{2}\right\rangle$. The law is valid in both two and three dimensions. See Ref. 13, for details. Setting $\mathbf{U}=\mathbf{0}$ in Eq. (3) reduces it to the standard third-order law for isotropic MHD turbulence, ${ }^{4}$ given herein as Eq. (2).

In order to carry out numerical tests, we now specialize to the case of $2 \mathrm{D}$ geometry. This will improve the statistical significance of our numerical results. Moreover, a fully three-dimensional treatment would necessarily have a less extensive inertial range.

Let us define $P_{3}^{ \pm}(\mathbf{r})=\left\langle\delta z_{k}^{\mp}\left|\delta z_{i}^{ \pm}\right|^{2}\right\rangle r_{k} / r$, where $\mathbf{r}$ is the separation vector. For isotropic MHD (with $\mathbf{U}=\mathbf{0}$ ), we will have $P_{3}^{ \pm}(r)=\left\langle\delta z_{r}^{\mp}\left|\delta z_{i}^{ \pm}\right|^{2}\right\rangle$. Choosing the integration "volume" to be a circle of radius $r$ means that Eq. (3) simplifies to

$$
P_{3}^{ \pm}(r)=-2 \epsilon^{ \pm} r,
$$

which is the $2 \mathrm{D}$ version of the third-order law, Eq. (2).

For anisotropic 2D MHD with the shear chosen to be constant, solenoidal, and in the $x$ direction, $\alpha=\mathrm{d} U_{y}(x) / \mathrm{d} x$, Eq. (3) becomes

$$
\begin{aligned}
& \int_{0}^{2 \pi} P_{3}^{ \pm}(\mathbf{r}) r \mathrm{~d} \theta+\int_{0}^{2 \pi} \delta U_{r}\left\langle\left|\delta z_{i}^{ \pm}\right|^{2}\right\rangle r \mathrm{~d} \theta \\
& \quad+2 \alpha \int_{0}^{r} \int_{0}^{2 \pi}\left\langle\delta z_{y}^{ \pm} \delta z_{x}^{\mp}\right\rangle r^{\prime} \mathrm{d} \theta \mathrm{d} r^{\prime}=-4 \pi r^{2} \epsilon^{ \pm},
\end{aligned}
$$

where $\theta$ is the angle $\mathbf{r}$ makes with the $x$ axis and $\delta U_{r}$ $=\delta \mathbf{U}(\mathbf{r}) \cdot \hat{\mathbf{r}}$. We can then define

$$
\begin{aligned}
& S^{ \pm}=-\frac{1}{2 \pi} \int_{0}^{2 \pi} P_{3}^{ \pm}(\mathbf{r}) \mathrm{d} \theta, \\
& S_{U}^{ \pm}=-\frac{1}{2 \pi} \int_{0}^{2 \pi} \delta U_{r}\left\langle\left|\delta z_{i}^{ \pm}\right|^{2}\right\rangle \mathrm{d} \theta, \\
& S_{\alpha}^{ \pm}=-\frac{\alpha}{\pi r} \int_{0}^{r} \int_{0}^{2 \pi}\left\langle\delta z_{y}^{ \pm} \delta z_{x}^{\mp}\right\rangle r^{\prime} \mathrm{d} \theta \mathrm{d} r^{\prime} .
\end{aligned}
$$

In terms of Eqs. (6)-(8), Eq. (5) can be rewritten as

$$
S^{ \pm}+S_{U}^{ \pm}+S_{\alpha}^{ \pm}=2 \epsilon^{ \pm} r \text {. }
$$

The second term $S_{U}^{ \pm}$is similar to the ordinary third-order structure function $S^{ \pm}$, but involves shear effects, and $S_{\alpha}^{ \pm}$is another term that depends on the shear and also the mixed second-order structure function of the fluctuations.

\section{STRATEGY AND NUMERICAL SIMULATIONS}

To test how the modified third-order law, Eq. (9), is influenced by these new terms, we now compare it to results obtained from turbulence simulation data. After first verifying that in isotropic 2D MHD the usual law of Eq. (2) holds, we move on to the case of central interest here, namely, when shear is present. We not only retain a periodic geometry but also impose a large-scale coherent velocity that is characterized by wide regions of constant shear.

We perform direct numerical simulations of turbulence in order to test the validity of Eq. (9). We numerically simulated forced 2D incompressible MHD turbulence, solving the following modified equations for the vector potential $a$ and vorticity $\omega$ :

$$
\begin{aligned}
& \partial_{t} \omega+(\mathbf{v}+\mathbf{U}) \cdot \nabla(\omega+\Omega)=\mathbf{b} \cdot \nabla j+\nu \nabla^{2}(\omega+\Omega)+F_{\omega}, \\
& \partial_{t} a+(\mathbf{v}+\mathbf{U}) \cdot \nabla a=\eta \nabla^{2} a+F_{a},
\end{aligned}
$$

where the magnetic field $\mathbf{b}=\nabla a \times \hat{\mathbf{z}}$, and the current density $j=-\nabla^{2} a$. The velocity is given by $\mathbf{v}=\nabla \psi \times \hat{\mathbf{z}}$, where $\nabla^{2} \psi=$ $-\omega$. The specified shear velocity is $\mathbf{U}=U_{y}(x) \hat{\mathbf{y}}$ with associated vorticity $\boldsymbol{\Omega}=\nabla \times \mathbf{U}$, and both are constant in time. Time $t$ is in units of nominal large-scale nonlinear (eddy turnover) times. In the present paper, we only study cases in which the kinetic viscosity $\nu$ and magnetic diffusivity $\eta$ are equal.

A fully dealiased, pseudospectral code with periodic boundary conditions is used. The external forces $F_{\omega}$ and $F_{a}$ are time dependent and are imposed to retain constant kinetic and magnetic energy in the first two wavenumber shells (1 $\leq k \leq 2)$. Moreover, each time step, the two forcings are chosen to be orthogonal (adjusting the phases of the Fourier 


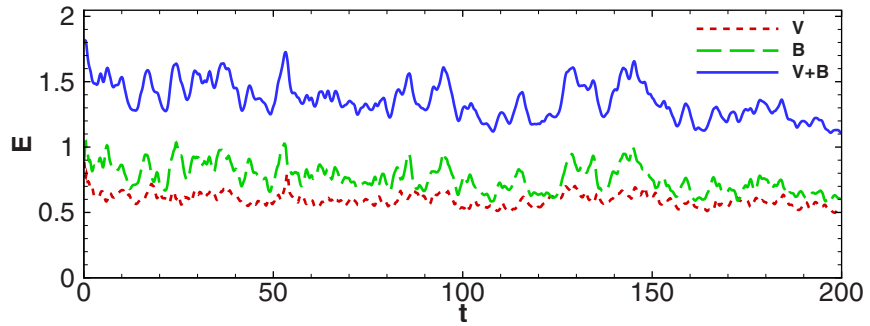

FIG. 1. (Color online) Time evolution of the kinetic (dotted red line), magnetic (dashed green line), and total (full blue line) energy for the isotropic MHD simulation (run 1). See Table I for more details on the simulation.

coefficients). For example, $\widetilde{\mathbf{F}}_{\omega} \cdot \widetilde{\mathbf{a}}=0$ for each wavevector, where denotes a quantity in Fourier space. This procedure keeps the cross helicity $\langle\mathbf{v} \cdot \mathbf{b}\rangle$ very small. Note that an arbitrary introduction of cross helicity, or substantial growth of it, would prevent development of a steady state because it suppresses the nonlinear terms. ${ }^{18}$

\section{A. Isotropic MHD turbulence}

For the first case, we simulate the 2D MHD system without imposed shear, setting $\mathbf{U}=\mathbf{0}$ in Eqs. (10) and (11). Figure 1 shows time evolution of total energy $\left\langle|\mathbf{v}|^{2}+|\mathbf{b}|^{2}\right\rangle / 2$ from a representative simulation. In investigating the third-order law, we use the data from $t=20$ to 200 , which we believe reasonably represents a steady state; snapshots of the full data are saved at a time interval of 1 . The time-averaged omnidirectional energy spectrum, averaged over more than 1800 energy spectra in this period, is shown in Fig. 2. For $k=4-30$, the averaged spectrum is found to be close to $k^{-5 / 3}$. This is an indication of the inertial range.

In order to better characterize the inertial range, and extract from the data the mean energy transfer rate, we now analyze the classical mixed third-order structure function as in Eq. (4). In Fig. 3 we show the scaling of $P_{3}^{ \pm}(r)$, with $2 \epsilon^{+} r$ plotted for comparison. (Since cross helicity is kept very small in the simulation, $\epsilon^{+} \simeq \epsilon^{-}$.) Both functions scale, in some range, $\propto r$. The results are consistent with the numerical results reported in Ref. 19 and validate Eq. (4) for the inertial

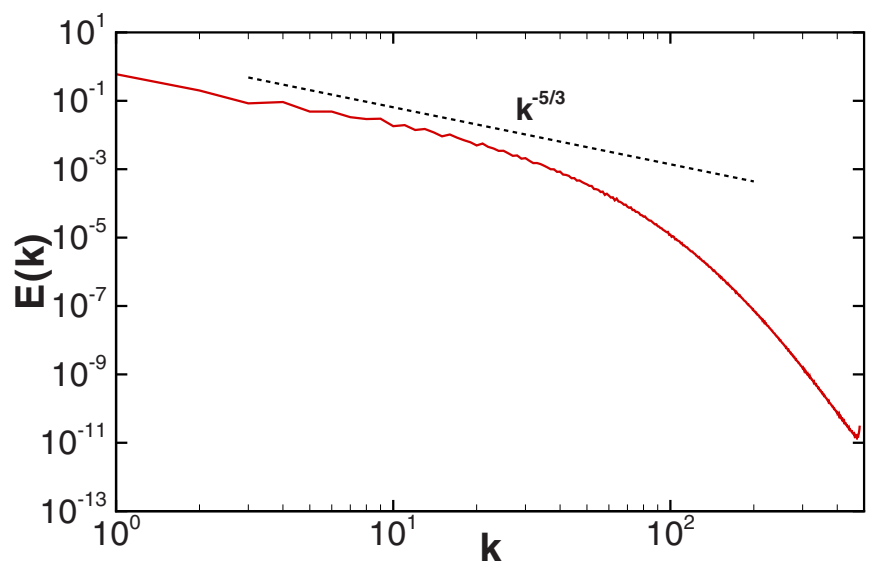

FIG. 2. (Color online) Time-averaged omnidirectional energy spectrum for the isotropic MHD simulation (full red line). For comparison, the Kolmogorov prediction $\sim k^{-5 / 3}$ is also shown (dashed black line).

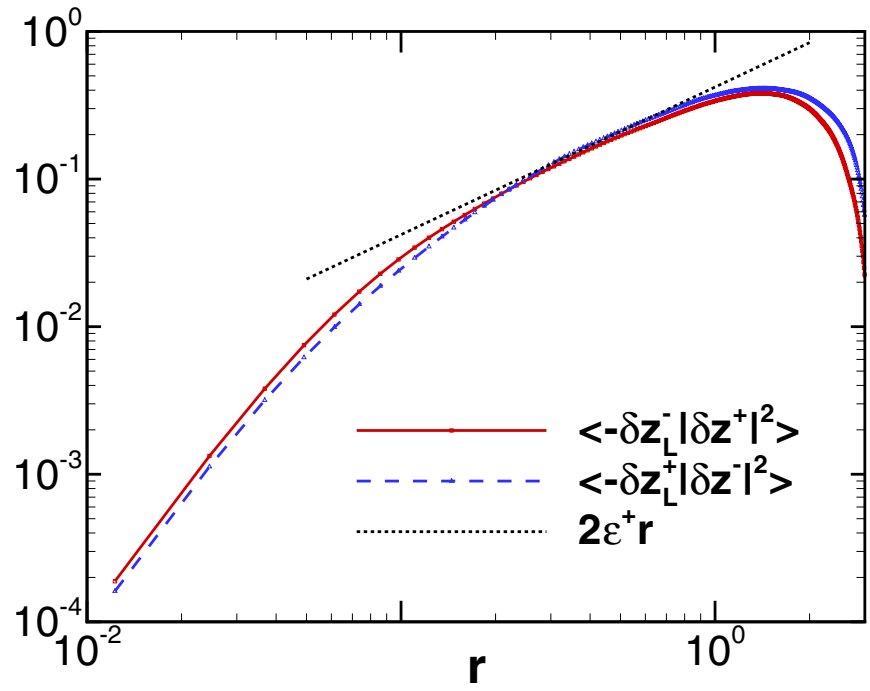

FIG. 3. (Color online) The scaling of mixed third-order structure functions for the isotropic MHD simulation (blue dashed and red solid line). The prediction of the inertial range scaling is also reported (dotted black line). See text for more details.

range. An alternative (and more sensitive) way to identify the inertial range is to look at the slope [or logarithmic derivative, $\left.\mathrm{d}\left(\ln P_{3}\right) / \mathrm{d}(\ln r)\right]$ of $P_{3}$ as a function of $r$, shown in Fig. 4. Clearly, a range where the slope is very close to 1 is present. At scales where the dissipation effects are dominant $(r<0.1)$, or where the increments are larger than the correlation length $(r>0.9)$, the third-order law is not valid.

Using Eq. (4), estimates for $\epsilon^{ \pm}$can be extracted from an observed linear scaling range of $P_{3}^{ \pm}(r)$. Based on the data in Fig. 3, we obtain $\epsilon^{+} \approx 0.21$ and $\epsilon^{-} \approx 0.22$. These values compare favorably with those calculated directly from the definition of the dissipation rates, $\epsilon^{ \pm} \equiv \nu\left\langle\left|\partial_{k} z_{i}^{ \pm}\right|^{2}\right\rangle=0.21$.

We note in passing the importance of using the signed third-order structure function in this analysis. Sometimes, when the amount of data available is insufficient to obtain good statistics, researchers have resorted to use of the unsigned third-order structure functions $\left\langle\left|\delta z_{L}^{\mp} \delta z_{i}^{ \pm 2}\right|\right\rangle$ instead of $\left\langle\delta z_{L}^{\mp}\left|\delta z_{i}^{ \pm}\right|^{2}\right\rangle .{ }^{19,20}$ While the unsigned quantities are useful for other purposes, they are not appropriate for the third-order law, as can be readily demonstrated. In Fig. 5 we show the scaling of $\left\langle\left|\delta z_{L}^{\mp} \delta z_{i}^{ \pm 2}\right|\right\rangle$ from our simulation. Although $\left\langle\left|\delta z_{L}^{\mp} \delta z_{i}^{ \pm}\right|^{2}\right\rangle$ exhibits a smooth scaling behavior in $r$, its be-

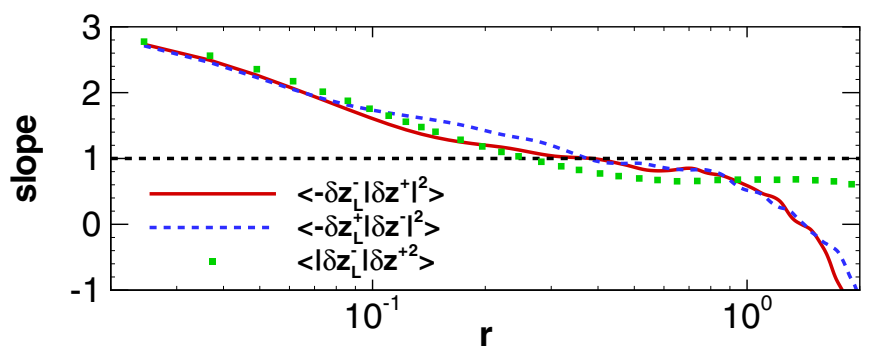

FIG. 4. (Color online) Slope as a function of the increment $r$ for the scaling of the mixed third-order structure functions (red solid and blue dashed lines). The slope of the third-order moment computed with the absolute value is also reported (filled green squares). 


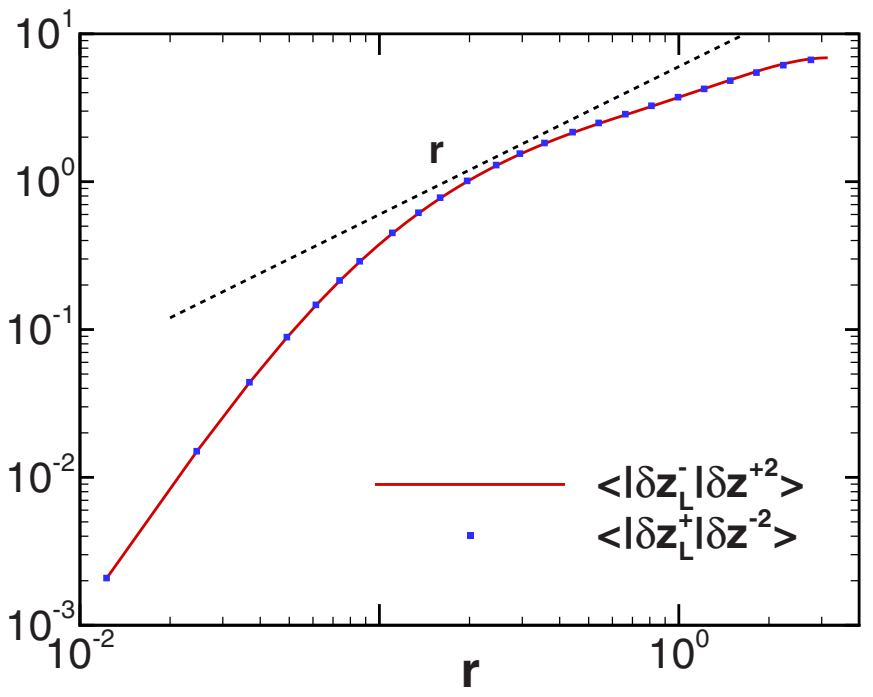

FIG. 5. (Color online) Scaling of mixed third-order structure functions with absolute sign. For comparison, the scaling $\sim r$ (black dashed line) is reported as well.

havior differs from that of $\left\langle\delta z_{L}^{\mp}\left|\delta z_{i}^{ \pm}\right|^{2}\right\rangle$, as the slope is smaller and the linear range is extended beyond the normal inertial range. The slope of $\left\langle\left|\delta z_{L}^{\mp} \delta z_{i}^{ \pm 2}\right|\right\rangle$ in its linear range is measured to be close to 0.8 , consistent with the results in Ref. 21 . This difference can be understood in the following way. Without the absolute value there are many terms that annihilate each other in the ensemble average, this cancellation effect is a genuine phenomenon of turbulence. Indeed, the third-order law [e.g., Eq. (4)] can be interpreted as being a consequence of the skewness in the distribution of the thirdorder moment. By taking the absolute value when calculating the structure function, this important feature of turbulence is discarded. In our analysis below we always employ signed moments.

\section{B. Anisotropic MHD turbulence with uniform shear}

We wish to test the above generalization of the MHD third-order law, described in Eqs. (6)-(9), by examining turbulence that evolves in the presence of a constant shear. To enable a numerical experiment of this type that employs an accurate and well-tested pseudospectral method (as with the isotropic case in Sec. III A), we adopt the following strategy. We simulate 2D MHD turbulence in periodic geometry, but impose a large-scale coherent velocity that is characterized by alternating large regions of constant shear. Specifically, we apply a large-scale velocity $U_{y}(x)$ with the profile shown in Fig. 6. In the same figure, we also show the corresponding large-scale shear profile $\Omega$. The constant shear occurs in two regions, with a shear rate $\pm \alpha$, being $\alpha \equiv \partial U_{y} / \partial x$. As can be seen two buffer regions between the shear flows are present, where the imposed shear changes sign. The parameters for the simulation are listed in Table I.

In analogy to the isotropic case, we run the simulation for a time that is sufficient to establish a near steady state. A large number of snapshots of the fluctuating fields are stored, and the analysis of the terms entering the modified third-
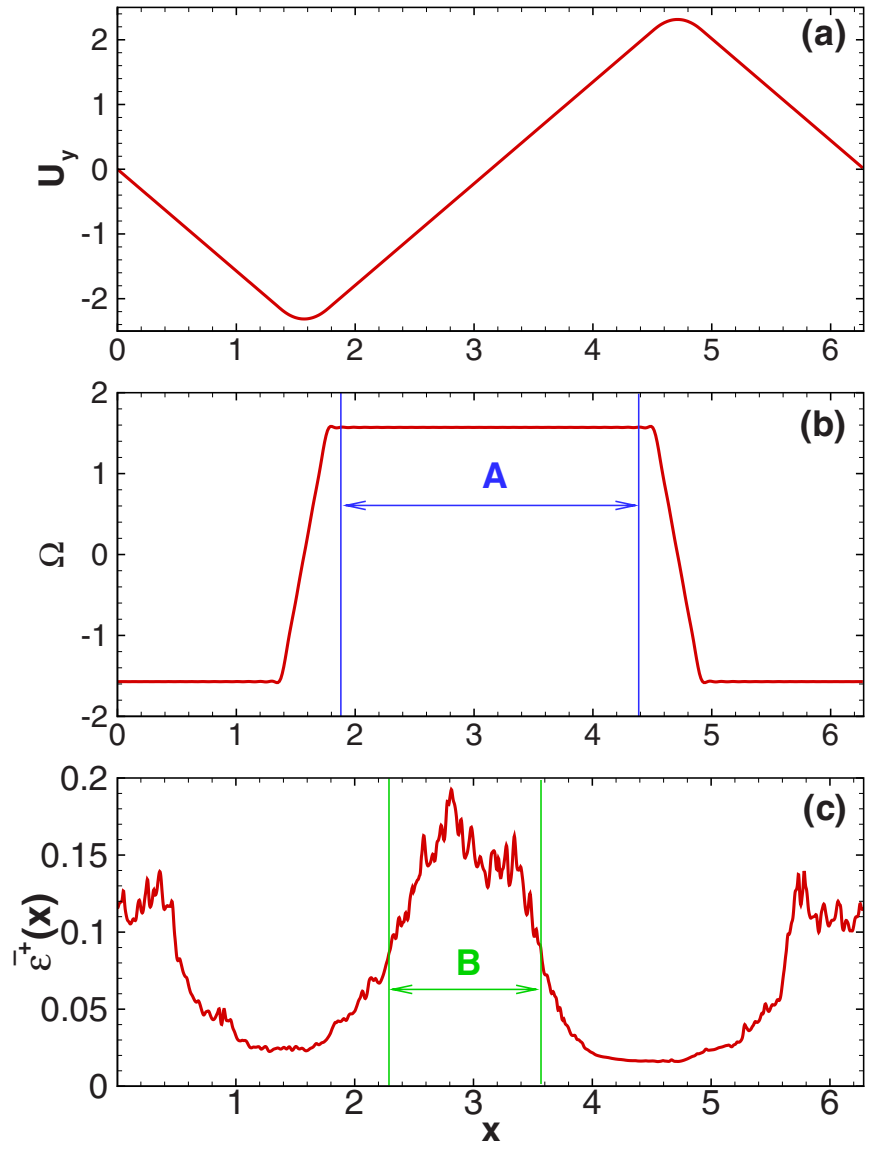

FIG. 6. (Color online) (a) Shear velocity profile $U_{y}(x)$; (b) shear profile of $\Omega$; (c) the averaged (in the $y$-direction) dissipation as a function of $x$. Clearly, the buffer regions at $x \sim 1.6$ and $x \sim 4.7$ introduce local anisotropy in the energy transfer rate. Regions A and B delimit quasihomogeneous parts of the domain.

order law Eq. (3) is carried out in the regions of nearly constant shear indicated in Fig. 6, avoiding the small buffer regions where the shear changes sign.

The evolution of kinetic energy, magnetic energy, and total energy, shown in Fig. 7, suggest that the simulation reached steady state after about $t=30$. For analysis, we have used the data from $t=30$ to 50 , which we identify as a quasisteady period. The (total) energy spectrum, averaged over direction and over this period of time, is shown in Fig. 8. The averaged spectrum is found to be close to $k^{-5 / 3}$ for $k=4-30$, which suggests an inertial range. Note that the modal energy spectrum is anisotropic and we revisit this below. These indicators suggest that this is a reasonable interval of data for carrying out an analysis of the shear-modified third-order law.

Figure 9 displays contours of electric current density. The field is strongly affected by the shear, with structures elongated in the $y$-direction. The correlation length $\ell$ is different along the $x$ and $y$ axes, with $\ell_{x} \approx 0.5$ and $\ell_{y} \approx 1.0$. Clearly the shear has rendered invalid the usual procedures employed for third-order analysis in isotropic turbulence.

The modal total energy spectrum (as a function of $k_{x}$ and $k_{y}$ ) is reported in Fig. 10, using a logarithmic scale. It is clear that the shear introduces strong anisotropy, with the spectrum "stretched" along the $k_{x}$ direction. The effect is mainly at low 
TABLE I. Parameters for the isotropic $(\alpha=0)$ and anisotropic simulations, where $r_{\mathrm{diss}}^{ \pm} \equiv\left(\nu^{3} / \epsilon^{ \pm}\right)^{1 / 4}$ are the Kolmogorov scales, and $\lambda_{T}^{ \pm} \equiv\left(\left\langle\left|\mathbf{z}^{ \pm}\right|^{2}\right\rangle /\left\langle\left|\nabla \times \mathbf{z}^{ \pm}\right|^{2}\right\rangle\right)^{1 / 2}$ are the Taylor microscales.

\begin{tabular}{ccccccc}
\hline \hline Run & Grid & $\nu=\eta$ & $\epsilon^{+} / \epsilon^{-}$ & $r_{\text {diss }}^{+} / r_{\text {diss }}^{-}$ & $\lambda_{T}^{+} / \lambda_{T}^{-}$ & $\alpha$ \\
\hline 1 & $1024^{2}$ & 0.001 & $0.21 / 0.21$ & $0.0083 / 0.0083$ & $0.12 / 0.11$ & 0 \\
2 & $2048^{2}$ & 0.0006 & $0.071 / 0.063$ & $0.0074 / 0.0077$ & $0.11 / 0.11$ & 1.57 \\
\hline \hline
\end{tabular}

wavenumbers. Large-scale shear induces a spectral anisotropy $^{22}$ similar to the case in which a uniform external magnetic field is imposed. ${ }^{23,24}$

In Fig. 11, we show the scaling of $S^{+}, S_{U}^{+}$, and $S_{\alpha}^{+}$using data from the positive shear region, with $2 \epsilon^{+} r$ plotted for comparison. Here $\epsilon^{+}=0.084$ is also evaluated only in the selected region. (Results for $S^{-}, S_{U}^{-}$, etc., are similar.) Although we can observe a short range where $S^{+}$scales $\propto r$, this range is much smaller than the isotropic case (see Fig. 3). If one was to attempt to use this linear scaling of $S^{+}$as a substitute for the full modified third-order law, Eq. (3), there is no assurance that the result would be relevant to the actual dissipation rate. However, one might be tempted to carry out this procedure if, for example, this were experimental data and the experimenters were unaware of the presence of the shear, and therefore assumed that Eq. (2) was valid. In fact, for this particular case the dissipation extracted from this scaling would be $\epsilon^{+} \approx 0.03$, which grossly underestimates the actual dissipation rate.

Even though we observe a very long range where $S_{U}^{+}$ $\propto r$, there is no reason that $S^{+}, S_{U}^{+}$, and $S_{\alpha}^{+}$should each have a (separate) scaling law; in fact, as obtained in Eq. (9), only the sum of these quantities obeys the scaling law. We can see evidence, in Fig. 11, that the modified third-order law produces a very good linear scaling. For the sum of the three terms, we get a much longer range of scaling $\propto r$, compared to any individual term, and the dissipation extracted from this scaling is $\epsilon^{+} \approx 0.1$, which only slightly overestimates the actual dissipation.

Although our simulations yield encouraging agreement with the third-order law modified for shear, they are only at modest Reynolds numbers. It follows that dissipative effects might be degrading the agreement. We now investigate whether better agreement is attainable by allowing for these effects. Recall that in deriving Eq. (3), a term involving vis-

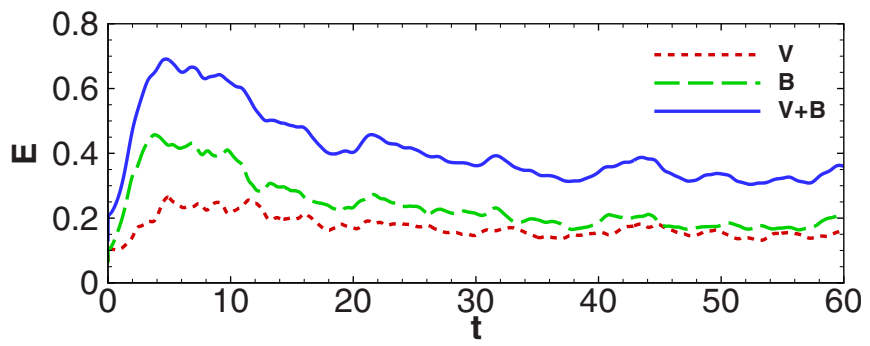

FIG. 7. (Color online) Time evolution of the kinetic (dotted red line), magnetic (dashed green line), and total energy (full blue line) for the shear MHD simulation (run 2). cosity was neglected by restricting $r$ to lie in the inertial range (see Ref. 4). In fact, this term is an additional contribution to the left side of Eq. (3), namely,

$$
-2 \nu \oint \frac{\partial}{\partial r_{k}}\left\langle\left|\delta z_{i}^{ \pm}\right|^{2}\right\rangle n_{k} \mathrm{~d} S_{r} .
$$

For 2D MHD, this viscous term can be written as

$$
S_{\nu}^{ \pm}=\frac{\nu}{\pi} \frac{\partial}{\partial r} \int_{0}^{2 \pi}\left\langle\left|\delta z_{i}^{ \pm}\right|^{2}\right\rangle \mathrm{d} \theta .
$$

Thus Eq. (9) changes to

$$
\Sigma^{ \pm} \equiv S^{ \pm}+S_{U}^{ \pm}+S_{\alpha}^{ \pm}+S_{\nu}^{ \pm}=2 \epsilon^{ \pm} r
$$

which is valid for all $r$ (much) less than the correlation length. To test this exact law, in Fig. 11 we also show the scaling of $\Sigma^{+}$. The result agrees with the theory quite well over the whole range, with $\Sigma^{+}$slightly bigger than $2 \epsilon^{+} r$.

In Fig. 12, we show the slopes of $S^{+}, S^{+}+S_{U}^{+}+S_{\alpha}^{+}$, and $\Sigma^{+}$. At very small scales (dissipation scales), the slopes of $S^{+}$ and $S^{+}+S_{U}^{+}+S_{\alpha}^{+}$are found to be very close to 3 . The slope of $S^{+}$is found to be decreasing quickly with separation $r$ increasing. However, there is little evidence for $S^{+}$having a region of constant slope. In contrast, we observe a quite long range where the slope of $S^{+}+S_{U}^{+}+S_{\alpha}^{+}$is nearly constant, although it tends to be slightly larger than the value of unity predicted by Eq. (9). This discrepancy can be viewed as being mainly caused by viscous effects (or that the inertial range is too short), as the slope of the viscous compensated total, $\Sigma^{+}$is found to be much closer to 1 .

The above analysis was performed using data from the positive shear region "A" (see Fig. 6), since $\Omega$ tends to be

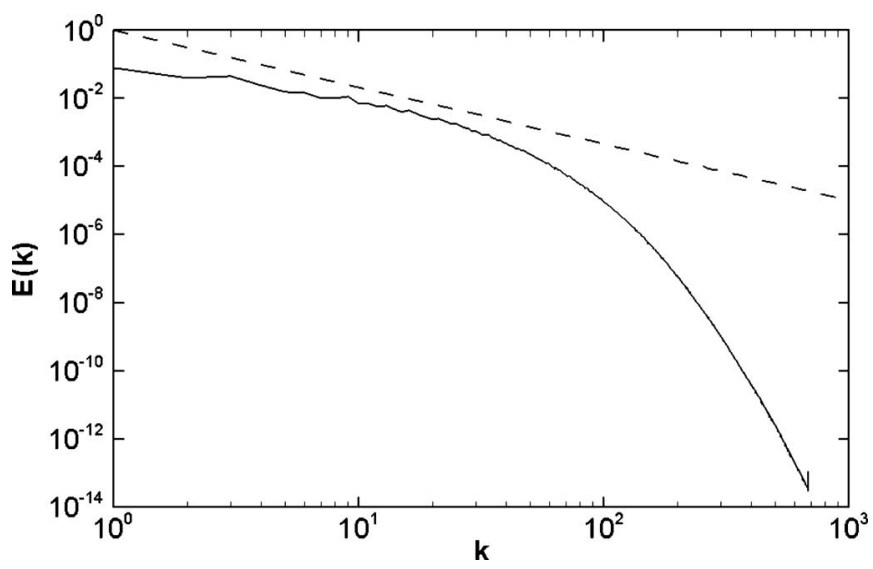

FIG. 8. Time-averaged omnidirectional energy spectrum for the shear MHD simulation; for comparison, $k^{-5 / 3}$ is plotted as a dashed line. 


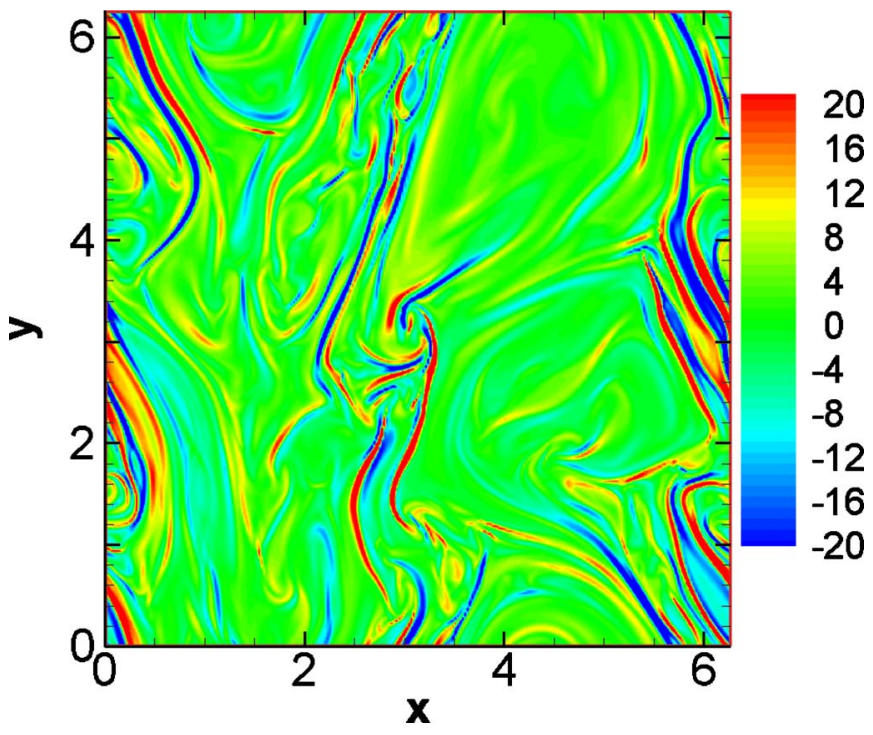

FIG. 9. (Color online) Color map of the magnetic current $j$ for the shear MHD simulation (run 2). The shear produces anisotropy, with the structures stretched along the $y$-direction. Scale (right) indicates values of the current density $j$.

quite constant in this region and homogeneity-a crucial assumption in deriving Eq. (3) - would be expected to be better satisfied there. Also plotted in Fig. 6 is the (time and $y$ ) averaged dissipation,

$$
\overline{\epsilon^{+}}(x)=\frac{1}{2 \pi T} \int_{0}^{T} \int_{0}^{2 \pi} \nu\left|\partial_{k} z_{i}^{+}\right|^{2} \mathrm{~d} y \mathrm{~d} t
$$

as a function of $x$. As we can see, the dissipation profile is not constant. Clearly, $\overline{\epsilon^{+}}(x)$ is bigger in the center region, but much smaller close to the buffer region, which does not satisfy homogeneity. For this reason, we have repeated the

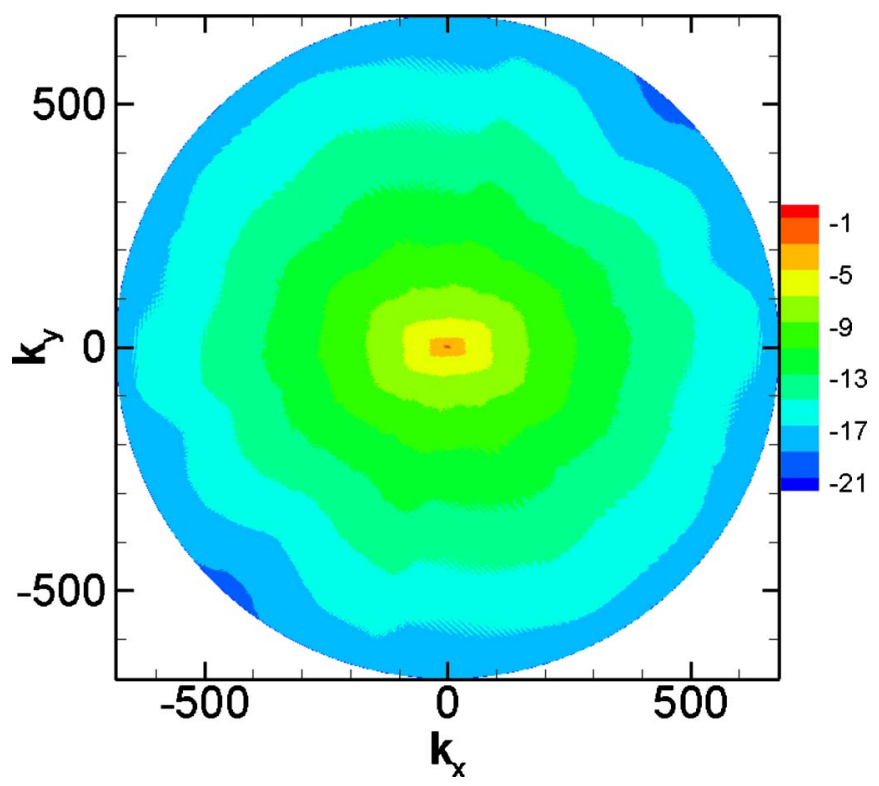

FIG. 10. (Color online) Modal total energy spectrum, showing the anisotropy that develops due to the presence of velocity shear. The logarithmic color scale (right) indicates the energy spectrum level in each region.

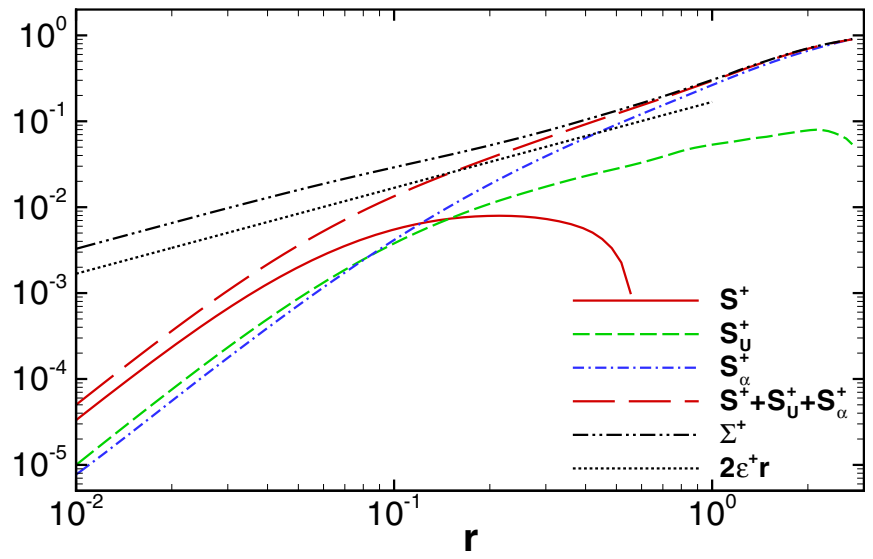

FIG. 11. (Color online) Scaling of the individual terms in the third-order law: $S^{+}$(full red line), $S_{U}^{+}$(dashed green line), and $S_{\alpha}^{+}$(dashed-dot blue line). Their sum $S^{+}+S_{U}^{+}+S_{\alpha}^{+}$(long-dashed red line), $\Sigma^{+}$(dashed-dot-dot black line), and the prediction given by Eqs. (9) and (14) (dotted black line) are also shown.

analysis using the data from region " $\mathrm{B}$," where we anticipate that homogeneity will be better satisfied.

In Fig. 13 we show the scaling of the individual thirdorder terms $S^{+}, S_{U}^{+}, S_{\alpha}^{+}$, their sum $S^{+}+S_{U}^{+}+S_{\alpha}^{+}$, and $\Sigma^{+}$using data from region "B." $2 \epsilon^{+} r$ is also plotted for comparison, where averaged dissipation $\epsilon^{+}=0.138$ in region " $\mathrm{B}$ " is used. Comparing Fig. 13 with Fig. 11, the biggest difference is that there is a much longer range in Fig. 13 where $S^{+}$scales with $r$, while in Fig. $11 S^{+}$falls off quickly with $r$. This is revealed more clearly in Fig. 14, which plots the slopes themselves. In contrast to Fig. 12, we observe a good range of scales where the slope of $S^{+}$is close to 1 . However, if we just use this scaling of $S^{+}$to predict $\epsilon^{+}$, we would still seriously underestimate the dissipation, as was also the case in Fig. 11. The point to emphasize is that unless one knows that shear is absent, scaling of $S^{+}(r)$ cannot be used in isolation to determine the dissipation rate.

\section{REYNOLDS DECOMPOSITION REVISITED}

In the previous sections we have discussed the thirdorder law for fluctuating quantities when a (uniform and constant) background shear flow is present. As Reynolds decomposition is widely used in turbulence studies, this seems to be a reasonable approach. For example, in Fig. 15 we show a typical signal of the fluctuation velocity component $u_{y}$, as

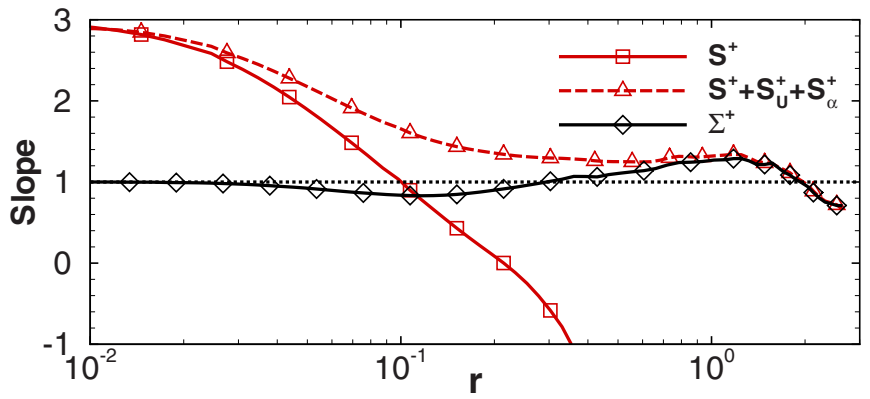

FIG. 12. (Color online) Slope of the mixed third-order structure functions as a function of the increment $r$ for run 2. See caption of Fig. 11. 


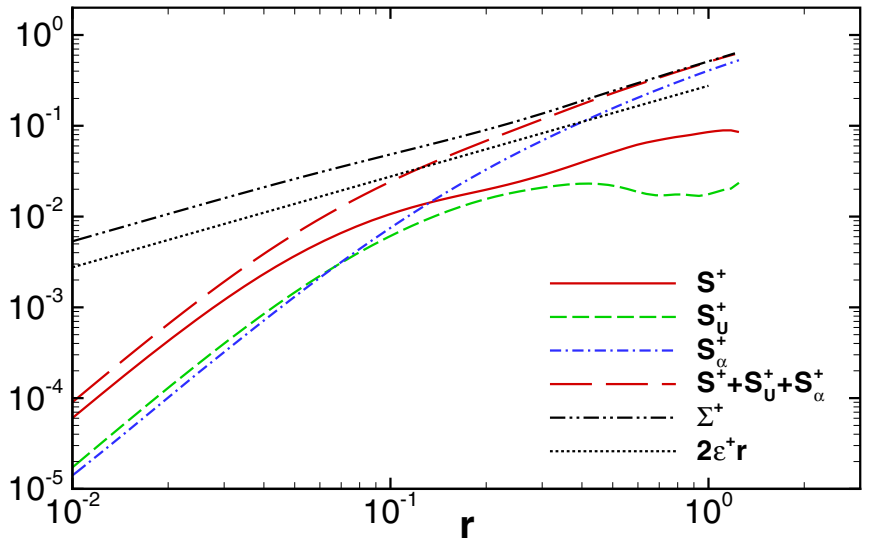

FIG. 13. (Color online) The scaling of the individual third-order terms $S^{+}$, $S_{U}^{+}$, and $S_{\alpha}^{+}$, their sum $S^{+}+S_{U}^{+}+S_{\alpha}^{+}$, and $\Sigma^{+}$, using data from region B. See caption of Fig. 11 for more details.

well as the full velocity component $u_{y}+U_{y}$, as a function of $x$ from Run 2 . With $U_{y}$ representing a nearly constant planar shear, it seems completely reasonable to remove that component of the mean flow, or equivalently, to detrend the dataset, prior to analyzing the fluctuating components. However, for the fluctuations defined in this standard (decomposed) way, we have seen that the third-order structure functions do not provide a simple account of the cascade rates. We therefore now examine a different approach. Specifically we will not attempt a separation of the large-scale shear flow from the fluctuations, instead we simply treat the velocity and magnetic fields in their entirety.

In the absence of a mean magnetic field $\mathbf{B}_{0}$, Eq. (7) in Ref. 13 can be rewritten as

$$
\begin{aligned}
\partial_{t}\left(\delta U_{i}+\delta z_{i}^{ \pm}\right)= & -\left(\delta U_{k}+\delta z_{k}^{\mp}\right) \partial_{k}^{\prime}\left(\delta U_{i}+\delta z_{i}^{ \pm}\right)-\left(z_{k}^{\mp}+U_{k}\right) \\
& \times\left(\partial_{k}^{\prime}+\partial_{k}\right)\left(\delta U_{i}+\delta z_{i}^{ \pm}\right)-\left(\partial_{i}^{\prime}+\partial_{i}\right) \delta P \\
& +\nu\left(\partial_{k}^{\prime} \partial_{k}^{\prime}+\partial_{k} \partial_{k}\right)\left(\delta U_{i}+\delta z_{i}^{ \pm}\right),
\end{aligned}
$$

with $\mathbf{U}$ a constant, uniform shear velocity.

Multiplying the previous equation by $2\left(\delta U_{i}+\delta z_{i}^{ \pm}\right)$and averaging yields

$$
\begin{aligned}
\partial_{t}\left\langle\left|\delta U_{i}+\delta z_{i}^{ \pm}\right|^{2}\right\rangle= & -\frac{\partial}{\partial r_{k}}\left\langle\left(\delta U_{k}+\delta z_{k}^{\mp}\right)\left|\delta U_{i}+\delta z_{i}^{ \pm}\right|^{2}\right\rangle \\
& +2 \nu \frac{\partial^{2}}{\partial r_{k}^{2}}\left\langle\left|\delta U_{i}+\delta z_{i}^{ \pm}\right|^{2}\right\rangle \\
& -4 \nu\left\langle\left|\partial_{k}\left(U_{i}+z_{i}^{ \pm}\right)\right|^{2}\right\rangle .
\end{aligned}
$$

In arriving at this expression we make use of $\partial_{k}\langle\bullet\rangle$ $=-\left(\partial / \partial r_{k}\right)\langle\bullet\rangle$ and $\partial_{k}^{\prime}\langle\bullet\rangle=\left(\partial / \partial r_{k}\right)\langle\bullet\rangle$. These latter relations follow from spatial homogeneity (i.e., translation invariance of the statistical properties), which can be considered for some systems to be an exact property or an approximation, e.g., in the case of a weakly inhomogeneous system.

The last term of Eq. (17) can be identified with the dissipation rates,

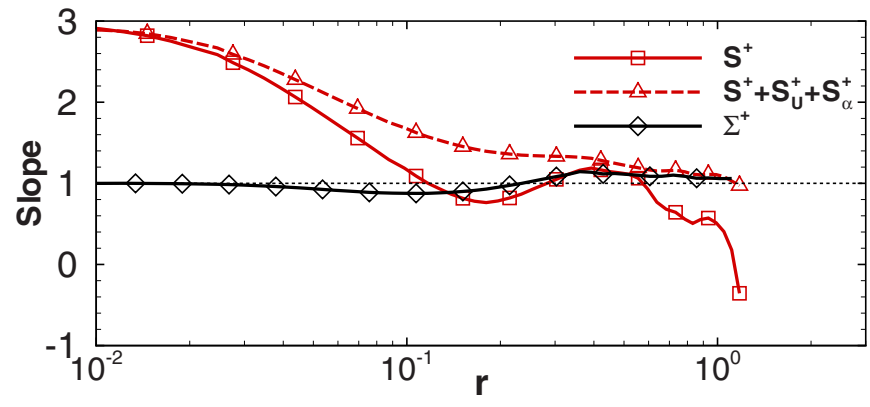

FIG. 14. (Color online) Slope for the scaling of mixed third-order structure function, and terms contributing to it, using data from region "B."

$$
\epsilon_{T}^{ \pm}=\nu\left\langle\left|\partial_{k}\left(U_{i}+z_{i}^{ \pm}\right)\right|^{2}\right\rangle,
$$

for the whole field $U_{i}+z_{i}^{ \pm}$. In the present study with constant shear velocity $\mathbf{U}$, it is not difficult to show $\epsilon_{T}^{ \pm}=\nu\left(\partial_{k} U_{i}\right)^{2}$ $+\epsilon^{ \pm}$. Under the hypothesis of steady-state turbulence and in the limit of vanishing viscosity $\nu \rightarrow 0$, Eq. (17) simplifies to

$$
\frac{\partial}{\partial r_{k}}\left\langle\left(\delta U_{k}+\delta z_{k}^{\mp}\right)\left|\delta U_{i}+\delta z_{i}^{ \pm}\right|^{2}\right\rangle=-4 \epsilon_{T}^{ \pm} .
$$

Specializing to 2D, integrating Eq. (19) in $\mathbf{r}$ over a circle of radius $r$, and using the divergence theorem on the left hand side yields

$$
S_{T}^{ \pm}=2 r \epsilon_{T}^{ \pm},
$$

where

$$
S_{T}^{ \pm}=-\frac{1}{2 \pi} \int_{0}^{2 \pi} \hat{r}_{k}\left\langle\left(\delta U_{k}+\delta z_{k}^{\mp}\right)\left|\delta U_{i}+\delta z_{i}^{ \pm}\right|^{2}\right\rangle \mathrm{d} \theta .
$$

To verify Eq. (20), we computed $S_{T}^{+}$for different separations $r$ using data from the anisotropic 2D MHD simulation. The results are plotted in Fig. 16. We have calculated $S_{T}^{+}$and $\epsilon_{T}^{+}$using data from the whole simulation domain (upper panel) and also only the data in subregion A (lower panel).

We see from the above results that examining the flow and magnetic fields directly - that is, not separating them into mean and fluctuating parts-gives a third-order structure function relation that is simple to interpret, and that is readily realized in the simulation data. The third-order total direction

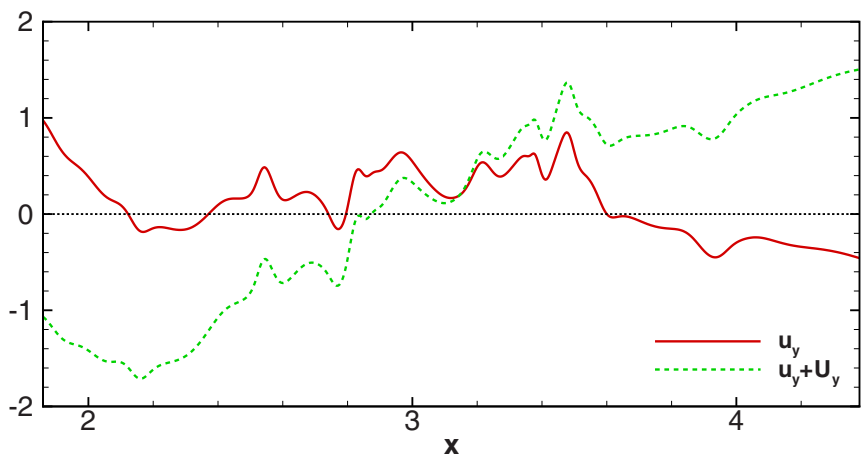

FIG. 15. (Color online) A typical fluctuation velocity component $u_{y}$ (full red line) as a function of $x$, extracted from the central region of the periodic domain in which there is a nearly constant shear (see Fig. 6), here compared with the total velocity component $u_{y}+U_{y}$ (dashed green line). 

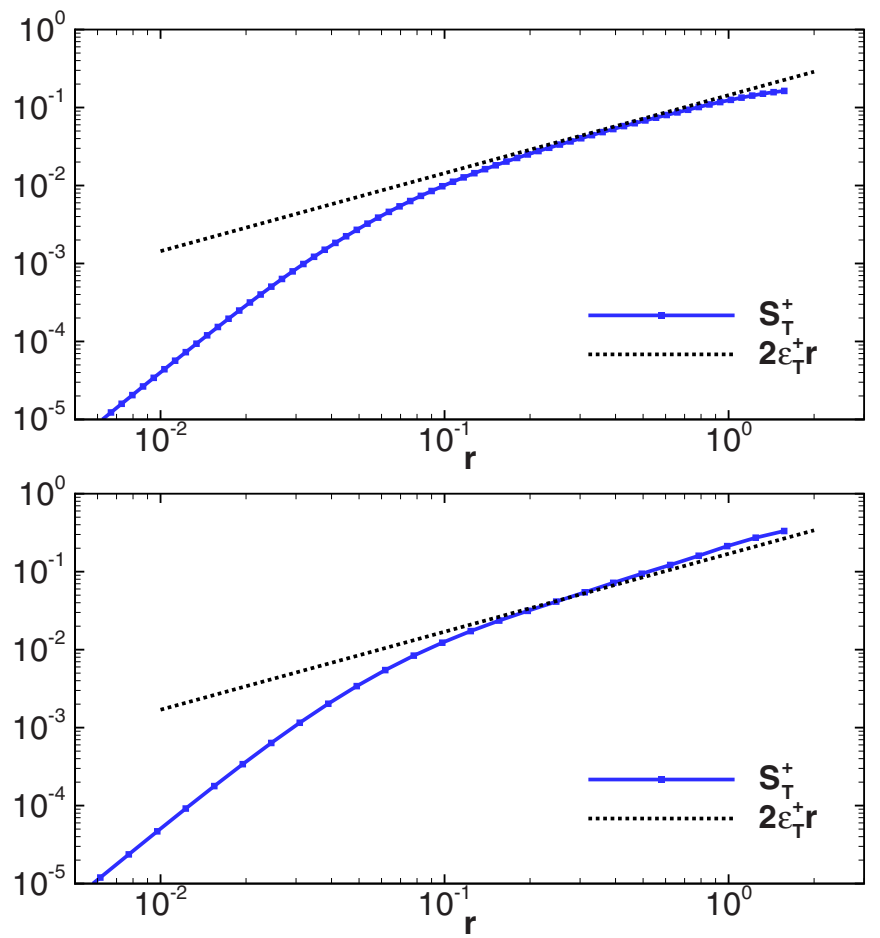

FIG. 16. (Color online) Total third-order moment $S_{T}^{+}$, defined in Eq. (21) (full blue line), using data from the whole field (top) and data from region A (bottom). For comparison, the prediction $2 r \epsilon_{T}^{+}$[see Eq. (20)] is plotted (dashed black line).

averaged energy flux vector is simply related to the steady total cascade rates of Eq. (18), much as the standard thirdorder law relates the similar quantities that are defined solely in terms of the fluctuation quantities [see Eq. (4)]. Familiar though these relations might appear to be, they are not standard in that they include the coherent, imposed shear flow on the same footing as the turbulent fluctuations. It is interesting to note that in Fig. 16 the third-order relation from the selected data region $\mathrm{A}$ in which the shear is approximately uniform does not work as well as the same relation computed over the full domain. Evidently, sampling the full coherent shear flow is required for an accurate third-order law when this approach is adopted.

\section{SUMMARY AND DISCUSSION}

We examined the mixed third-order Elsässer structure functions for MHD turbulence, incorporating a constant sheared velocity field in addition to homogeneous fluctuations, under a set of assumptions that parallels those used in standard turbulence theory to derive the Kolmogorov 4/5 law. In analogy to the findings of Casciola et al. ${ }^{14}$ and Lindborg $^{25}$ for HD, we find that a law can be obtained for stationary homogeneous turbulence that relates third-order structure functions and dissipation, but which also involves additional terms. For MHD with an imposed constant shear, there are shear related terms that appear in this modified third-order law, as in the HD case.

The 2D simulations that we carried out to demonstrate the new relationship were designed to enable high statistical weight tests to be done using an accurate pseudospectral code. We found that the modified relationship $S^{ \pm}+S_{U}^{ \pm}+S_{\alpha}^{ \pm}$ $=-2 \epsilon^{ \pm} r$ is well satisfied in the sense that the sum of the three structure function terms scales linearly with separation in the inertial range, even though the slope is found to be slightly larger than 1. We believe this steeper slope is caused by viscous effects, or, to put it another way, the Reynolds numbers of the simulations are not large enough to support asymptotic results for which the viscous effects are negligible. Moreover, retaining the viscous term $S_{\nu}^{ \pm}$in the derivation of the (modified) third-order law leads to the relation $S^{ \pm}+S_{U}^{ \pm}+S_{\alpha}^{ \pm}+S_{\nu}^{ \pm}=-2 \epsilon^{ \pm} r$, which is found to be valid over the whole range from the dissipation scale to the integral scale, with its slope very close to 1 .

We further examined the third-order structure functions for a periodic shear flow by analyzing the imposed coherent flow and the fluctuations together, instead of using the usual decomposition. While somewhat appealing intuitively, this procedure departs from the standard approaches, exemplified by Reynolds decomposition, in which mean flows are removed by detrending, filtering, or subtracting an average value, prior to analyzing the remaining fluctuations as the "turbulence." We carried out this alternative examination both analytically and in the simulation data. The conclusion emerges in both cases, that the third-order relation is restored to a simple form in the presence of large-scale coherent shear, if the coherent modes and the fluctuations are grouped together as a single entity. When analyzed in this way, the familiar anisotropic form of the third-order law is restored.

The implications of the above results for applications such as the solar wind are intriguing. A number of authors have examined third-order structure functions in the available interplanetary magnetic field datasets. Some linear scaling of third-order structure functions is observed, ${ }^{6}$ even though isotropy is not fully satisfied in the solar wind, ${ }^{26-28}$ due to the presence of a strong large-scale magnetic field. ${ }^{23,24}$ Other recent studies ${ }^{7,29}$ have also extended the treatment of the third-order MHD laws to a form in which spectral anisotropy can be accommodated. In particular, MacBride et $\mathrm{al}^{7}$ model the solar wind magnetic fluctuations as either an isotropic or a nonisotropic model (a mixture of one-dimensional and 2D fluctuations) when they evaluate heating rates using the homogeneous third-order law. However, their formulation as well as other similar studies (e.g., Refs. 6, 8, and 29) have so far maintained the assumption that the large-scale magnetic field and plasma flows are uniform. This eliminates by assumption the possibility that large-scale shear can enter the balance between cascade and dissipation, as it does in the Casciola HD study and its antecedents. ${ }^{14,25}$

The present results are complementary to these and may provide some guidance concerning selection and preparation of datasets for third-order analysis. Our results suggest that when fluctuations are separated from the mean flows and magnetic fields, the results may be affected by large-scale shear, and moreover data selection has only a limited influence on improvement of the results. In contrast, the novel procedure suggested above, of carrying out the analysis without separation into mean fields and fluctuations, appears to work much better and eliminates the need to take into account several new terms in the third-order relationships. 
Lacking details concerning preparation of datasets in the above mentioned observational analyses, we cannot comment further on the results. However, we do suggest for future studies that the issues raised above be considered. Finally, we remark that similar simplifications may be possible for HD third-order analysis, but pursuing this is beyond the scope of the present paper.

\section{ACKNOWLEDGMENTS}

This research supported in part by the NSF Solar Terrestrial Program under Grant No. ATM0539995 and by NASA under the Heliophysics Theory Program Grant No. NNX08AI47G.

${ }^{1}$ A. N. Kolmogorov, C. R. Acad. Sci. URSS 32, 16 (1941) [reprinted in Proc. R. Soc. London, Ser. A 434, 15 (1991)].

${ }^{2}$ A. S. Monin and A. M. Yaglom, Statistical Fluid Mechanics (MIT, Cambridge, 1975), Vol. 2.

${ }^{3}$ U. Frisch, Turbulence (CUP, Cambridge, 1995).

${ }^{4}$ H. Politano and A. Pouquet, Geophys. Res. Lett. 25, 273, doi:10.1029/ 97GL03642 (1998).

${ }^{5}$ H. Politano and A. Pouquet, Phys. Rev. E 57, R21 (1998).

${ }^{6}$ L. Sorriso-Valvo, R. Marino, V. Carbone, A. Noullez, F. Lepreti, P. Veltri, R. Bruno, B. Bavassano, and E. Pietropaolo, Phys. Rev. Lett. 99, 115001 (2007).

${ }^{7}$ B. T. MacBride, C. W. Smith, and M. A. Forman, Astrophys. J. 679, 1644 (2008).

${ }^{8}$ R. Marino, L. Sorriso-Valvo, V. Carbone, A. Noullez, R. Bruno, and B. Bavassano, Astrophys. J. 677, L71 (2008).

${ }^{9}$ C. W. Smith, J. E. Stawarz, B. J. Vasquez, M. A. Forman, and B. T. MacBride, Phys. Rev. Lett. 103, 201101 (2009); J. J. Podesta, M. A. Forman, and C. W. Smith, Phys. Plasmas 14, 092305 (2007).
${ }^{10}$ T. de Kármán and L. Howarth, Proc. R. Soc. London, Ser. A 164, 192 (1938).

${ }^{11}$ M. Hossain, P. C. Gray, D. H. Pontius, Jr., W. H. Matthaeus, and S. Oughton, Phys. Fluids 7, 2886 (1995).

${ }^{12}$ B. Breech, W. H. Matthaeus, J. Minnie, J. W. Bieber, S. Oughton, C. W. Smith, and P. A. Isenberg, J. Geophys. Res. 113, A08105, doi:10.1029/ 2007JA012711 (2008).

${ }^{13}$ M. Wan, S. Servidio, S. Oughton, and W. H. Matthaeus, Phys. Plasmas 16, 090703 (2009).

${ }^{14}$ C. M. Casciola, P. Gualtieri, R. Benzi, and R. Piva, J. Fluid Mech. 476, 105 (2003).

${ }^{15}$ R. J. Hill, J. Fluid Mech. 353, 67 (1997).

${ }^{16} \mathrm{H}$. Tennekes and J. L. Lumley, A First Course in Turbulence (MIT, Cambridge, 1972).

${ }^{17}$ D. J. McComas, L. Barraclough, H. O. Funsten, J. T. Gosling, E. SantiagoMuñoz, R. M. Skoug, B. E. Goldstein, M. Neugebauer, P. Riley, and A. Balogh, J. Geophys. Res. 105, 10419, doi:10.1029/1999JA000383 (2000).

${ }^{18}$ R. H. Kraichnan, Phys. Fluids 8, 1385 (1965).

${ }^{19}$ L. Sorriso-Valvo, V. Carbone, A. Noullez, H. Politano, A. Pouquet, and P. Veltri, Phys. Plasmas 9, 89 (2002).

${ }^{20}$ H. Politano, A. Pouquet, and V. Carbone, Europhys. Lett. 43, 516 (1998).

${ }^{21}$ D. Biskamp and E. Schwarz, Phys. Plasmas 8, 3282 (2001).

${ }^{22}$ D. A. Roberts, M. L. Goldstein, W. H. Matthaeus, and S. Ghosh, J. Geophys. Res. 97, 17115, doi:10.1029/92JA01144 (1992).

${ }^{23}$ J. V. Shebalin, W. H. Matthaeus, and D. Montgomery, J. Plasma Phys. 29, 525 (1983).

${ }^{24}$ S. Oughton, E. R. Priest, and W. H. Matthaeus, J. Fluid Mech. 280, 95 (1994).

${ }^{25}$ E. Lindborg, J. Fluid Mech. 326, 343 (1996).

${ }^{26}$ J. W. Belcher and L. Davis, Jr., J. Geophys. Res. 76, 3534, doi:10.1029/ JA076i016p03534 (1971).

${ }^{27}$ W. H. Matthaeus, M. L. Goldstein, and D. A. Roberts, J. Geophys. Res. 95, 20673, doi:10.1029/JA095iA12p20673 (1990).

${ }^{28}$ J. W. Bieber, W. Wanner, and W. H. Matthaeus, J. Geophys. Res. 101, 2511, doi:10.1029/95JA02588 (1996).

${ }^{29}$ J. E. Stawarz, C. W. Smith, B. J. Vasquez, M. A. Forman, and B. T. MacBride, Astrophys. J. 697, 1119 (2009). 